\title{
Predicting lymph node metastases in early gastric cancer: radical resection or organ-sparing therapy?
}

\author{
Alexander R. Novotny and Christoph Schuhmacher \\ Department of Surgery, Technische Universität München, Ismaninger Str. 22, 81675 Munich, Germany
}

Diagnosis and therapy of early gastric cancer (EGC) experienced a gradual change during the last decade owing to better diagnostic techniques and new treatment strategies. Between Western countries and Japan opposing trends in the surgical therapy of early gastric cancer have been observed in recent years. While Western surgeons increasingly use more radical therapies in the treatment of curable gastric cancer, Japanese surgeons who were traditionally focused on radicality have started using more conservative treatment methods such as endoscopic mucosal resection (EMR), endoscopic submucosal dissection (ESD), laparoscopic wedge resection or function-preserving gastrectomies in EGC. This change is a result of the higher detection rate of gastric cancer at an early stage of the disease owing to the national screening program carried out in Japan and the constant struggle for the individualization of gastric cancer therapy for the sake of a lower morbidity and better quality of life. In Western countries EGC is still a rare disease because almost half of the patients are already harboring stage III or IV disease when they are admitted to treatment.

The presence of lymph node metastases is recognized as the most significant prognostic factor for survival in patients with EGC [1] and usually constitutes the watershed between radical surgery and limited, organoriented therapy. A prerequisite for limited radicality in the therapy for EGC is therefore accurate tumor staging, predicting lymph-node involvement. To date, the probability of tumor-positive lymph nodes in EGC has been estimated on the basis of macroscopicendoscopic tumor characteristics and biopsy findings such as tumor differentiation and depth of invasion. Numerous newer studies have included further variables using immunohistochemistry with the aim of defining new patient subgroups with EGC who are potential candidates for limited surgery or EMR/ESD.

Offprint requests to: A.R. Novotny
It is in this context that the work of Kwee and Kwee [2] has to be seen. In their article they describe the results of a meta-analysis investigating the association between certain patient- and tumor-related parameters as well as molecular markers in EGC and the presence of lymph node metastases.

Generally, in about $8 \%-20 \%$ of EGC cases, depending on the reported series, lymph-node metastases can be expected [3-5].

Shimada et al. reported an incidence of lymph node metastases from mucosal (M) and submucosal (SM) tumors of $2.5 \%$ and $20.2 \%$, respectively [6]. These findings correspond well with those of Gotoda et al. [1], who found lymph node metastases in $2.2 \%$ of intramucosal cancers and in $17.9 \%$ of cancers invading the submucosa. In the latter, the degree of submucosal penetration was of great importance: while only $8.8 \%$ of tumors of submucosa 1 (SM1) grade $(<500 \mu \mathrm{m}$ from the muscularis mucosa) were node positive, $23.7 \%$ of tumors of submucosa 2 (SM2) grade ( $\geq 500 \mu \mathrm{m}$ from the muscularis mucosa) showed lymph node involvement [1]. Because direct detection of positive lymph nodes with accurate sensitivity is not possible yet, the indirect estimation of the presence of tumor-positive lymph nodes is of major importance.

The indirect assessment of lymph node metastases in EGC is still carried out by endoscopy and biopsy of the primary lesion. The extended indications for EMR or ESD as defined by the Treatment guidelines for gastric cancer in Japan [7] are based on findings provided by these examinations, namely (1) differentiated type mucosal cancer without ulcer and larger than $2 \mathrm{~cm},(2)$ differentiated mucosal cancer with ulcer and $3 \mathrm{~cm}$ or smaller, and (3) undifferentiated type mucosal cancer without ulcer and $2 \mathrm{~cm}$ or smaller. According to the guidelines, additional lymph node resection is not necessary if lymphovascular invasion is absent and the depth of wall invasion is not deeper than SM1 in the biopsy specimen $[7,8]$. While some authors propose to 
further extend these criteria [1], others advise against the use of the extended criteria, recommending only local treatment for mucosal cancers without ulcer and differentiated type mucosal cancers with ulcers smaller than $2 \mathrm{~cm}$ [9]. Once the limited treatment has been carried out, care should be taken in the processing and evaluation of the histological specimen. If it is discovered during the histologic workup that any of the abovementioned criteria are violated, the initial limited procedure has to be followed by a more radical surgical resection.

Complementary to EMR and ESD, a multitude of limited surgical procedures exist, including pyloruspreserving distal gastrectomy [10], segmental gastrectomy [11], proximal gastrectomy [12], limited resection of the gastric cardia [13], and partial gastrectomy [14]. Distinct guidelines have been developed for the use of each procedure. Guidelines for the required lymphadenectomy when a limited surgical procedure is used are also provided by the Treatment guidelines for gastric cancer in Japan [7]. Mucosal tumors without lymph node metastases and submucosal tumors of differentiated histological type and less than $1.5 \mathrm{~cm}$ in diameter without lymph node metastases can be operated on using minimal lymph node dissection of the D1 nodes + No. 7 and No. 8 a nodes in lesions located in the lower third of the stomach. Tumors of more than $2 \mathrm{~cm}$ in diameter with $\mathrm{N} 1$ node metastases or a tumor with $\mathrm{N} 2$ lymph node metastases require a standard resection (total gastrectomy or two-thirds of the stomach) with D2 dissection. All other early gastric cancers with N0 or N1 status can be operated on using minimal lymph node dissection of the D1 nodes + No. 7, 8a, and 9 nodes.

The above-mentioned guidelines have been devised on the basis of vigorous retrospective studies and databases containing several hundreds and even thousands of EGC patients who have undergone surgery that included lymphadenectomy. The guidelines help to identify patients who qualify for limited treatment, and they are widely used in Japan, where endoscopists have the required expertise owing to the large number of EGCs detected. While the guidelines for EMR and ESD are based on criteria that are easily assessable during the staging process, the guidelines for surgical treatment require the preoperative detection of lymph node metastases. It is still impossible, however, to accurately detect cancer-positive lymph nodes by imaging techniques such as endoscopic ultrasound (EUS) or computed tomography (CT) $[15,16]$. For this reason, multiple patient- and tumor-related variables are investigated as predictors of lymph node involvement, especially in EGC with invasion of the submucosa [17].

The Treatment guidelines for gastric cancer in Japan [7] provide a sound basis for identifying patient sub- groups eligible for limited treatment of EGC in the hands of experienced Japanese specialists. In a country like Japan with a high incidence of EGCs (up to 70\% of all detected gastric cancers), one can rely on this large pool of experience. The guidelines have their limitations, however, in case of EGC invading the submucosa, because eventual lymph node metastases are not detectable with a high enough sensitivity by the currently available preoperative diagnostic tools.

In Western countries the incidence of EGC is about $15 \%$ owing to the lack of institutionalized screening programs and a population less alert to the importance of such screening. Because Western endoscopists rarely encounter gastric cancer in an early stage, their expertise in classifying EGCs according to the Japanese guidelines is limited. Additionally, the availability of endoscopic ultrasound is limited to specialized centers, which, however, do not perform the majority of endoscopies. Thus, simple and dependable factors are needed to evaluate a tumor before a lymphatic spread has taken place.

Therefore the information provided in the article by Kwee and Kwee [2] is of importance because it evaluates and sums up the results of multiple studies dealing with this issue. In their discussion the authors present the outlook on a model for predicting lymph node metastases in EGC based on the variables found in their study after evaluating them on an independent set of patients and eliminating confounding variables by multivariate analysis. The real value of the work by Kwee and Kwee lies in the collection of these not-yetestablished but promising variables providing the basis for further investigations. A model like the one proposed will certainly be awaited with anticipation by everyone involved in gastric cancer treatment.

Until then we have to continue using the alreadyestablished criteria which set forth a considerable amount of experience for their proper use. If in doubt one should always favor a more radical approach including lymphadenectomy over a minimally invasive therapy with the risk of tumor progression.

\section{References}

1. Gotoda T, Yanagisawa A, Sasako M, Ono H, Nakanishi Y, Shimoda T, et al. Incidence of lymph node metastasis from early gastric cancer: estimation with a large number of cases at two large centers. Gastric Cancer 2000;3:219-25.

2. Kwee RM, Kwee TC. Predicting lymph node status in early gastric cancer. Gastric Cancer 2008;11:134-148.

3. Borie F, Plaisant N, Millat B, Hay JM, Fagniez PL. Appropriate gastric resection with lymph node dissection for early gastric cancer. Ann Surg Oncol 2004;11:512-7.

4. Kikuchi S, Katada N, Sakuramoto S, Kobayashi N, Shimao H, Watanabe M, et al. Survival after surgical treatment of early gastric cancer: surgical techniques and long-term survival. Langenbecks Arch Surg 2004;389:69-74. 
5. Kunisaki C, Akiyama H, Nomura M, Matsuda G, Otsuka Y, Ono $\mathrm{H}$, et al. Surgical outcomes for early gastric cancer in the upper third of the stomach. J Am Coll Surg 2005;200:15-9.

6. Shimada S, Yagi Y, Honmyo U, Shiomori K, Yoshida N, Ogawa M. Involvement of three or more lymph nodes predicts poor prognosis in submucosal gastric carcinoma. Gastric Cancer 2001;4:54-9.

7. Japanese Gastric Cancer Association. Treatment guidelines for gastric cancer in Japan (in Japanese). 2nd ed. Tokyo: Kanehara; 2004.

8. Abe N, Sugiyama M, Masaki T, Ueki H, Yanagida O, Mori T, et al. Predictive factors for lymph node metastasis of differentiated submucosally invasive gastric cancer. Gastrointest Endosc 2004;60:242-5.

9. Ishikawa S, Togashi A, Inoue M, Honda S, Nozawa F, Toyama $\mathrm{E}$, et al. Indications for EMR/ESD in cases of early gastric cancer: relationship between histological type, depth of wall invasion, and lymph node metastasis. Gastric Cancer 2007;10:35-8.

10. Sawai K, Takahashi T, Fujioka $T$, Minato $H$, Taniguchi $H$, Yamaguchi T. Pylorus-preserving gastrectomy with radical lymph node dissection based on anatomical variations of the infrapyloric artery. Am J Surg 1995;170:285-8.

11. Ohwada S, Nakamura S, Ogawa T, Izumi M, Tanahashi Y, Sato $\mathrm{Y}$, et al. Segmental gastrectomy for early cancer in the midstomach. Hepatogastroenterology 1999;46:1229-33.
12. Furukawa H, Hiratsuka M, Imaoka S, Ishikawa O, Kabuto T, Sasaki, et al. Limited surgery for early gastric cancer in cardia. Ann Surg Oncol 1998;5:338-41.

13. Stein HJ, Feith M, Bruecher BL, Naehrig J, Sarbia M, Siewert JR. Early esophageal cancer: pattern of lymphatic spread and prognostic factors for long-term survival after surgical resection. Ann Surg 2005;242:566-73.

14. Seto Y, Nagawa H, Muto Y, Kaizaki S, Kitayama J, Muto T. Preliminary report on local resection with lymphadenectomy for early gastric cancer. Br J Surg 1999;86:526-8.

15. Polkowski M, Palucki J, Wronska E, Szawlowski A, NasierowskaGuttmejer A, Butruk E. Endosonography versus helical computed tomography for locoregional staging of gastric cancer. Endoscopy 2004;36:617-23.

16. Tsendsuren T, Jun SM, Mian XH. Usefulness of endoscopic ultrasonography in preoperative TNM staging of gastric cancer. World J Gastroenterol 2006;12:43-7.

17. An JY, Baik YH, Choi MG, Noh JH, Sohn TS, Kim S. Predictive factors for lymph node metastasis in early gastric cancer with submucosal invasion: analysis of a single institutional experience. Ann Surg 2007;246:749-53. 